\title{
Concurrent Persistent Cryptococcosis and Mycobacterium avium Complex Infections in a Patient With Human Immunodeficiency Virus
}

\author{
Abdulaziz U. Joury, MD, ${ }^{1,2}$ Mona Alshehri, MD, ${ }^{3}$ Mustafa Alhasan, MD ${ }^{4}$ \\ ${ }^{1}$ Department of Internal Medicine, Ochsner Clinic Foundation, New Orleans, LA ${ }^{2}$ King Salman Heart Center, King Fahd Medical City, Riyadh, \\ Saudi Arabia ${ }^{3}$ Department of Ophthalmology, King Saud University, Riyadh, Saudi Arabia ${ }^{4}$ Department of Radiology, Ochsner Clinic \\ Foundation, New Orleans, LA
}

\begin{abstract}
Background: Fungal infections are known for their chronicity and latency and are difficult to eradicate. The pathophysiology of these invading pathogens and the resulting alteration of the immune system are not fully understood. Fungal meningitis is associated with significant rates of morbidity and mortality, especially among immunocompromised patients. Cryptococcosis, an infection secondary to the fungus Cryptococcus, is one of the most important opportunistic infections among patients with human immunodeficiency virus (HIV), and expediting treatment is crucial.

Case Report: We report the case of a 28-year-old male with HIV who had a simultaneous co-infection with cryptococcal meningitis and Mycobacterium avium complex (MAC). The patient required a 2-week induction phase of antifungal medication with amphotericin B and flucytosine. Despite aggressive initial therapy and the patient's significant clinical improvement, his radiologic findings and lumbar puncture showed persistent cryptococcal infection.

Conclusion: Concurrent infection with cryptococcosis and MAC is extremely rare even in immunosuppressed patients. In our case, the concurrent infection was associated with a prolonged course of therapy during the induction phase for cryptococcosis.
\end{abstract}

Keywords: Cryptococcosis, HIV, meningitis-cryptococcal, Mycobacterium avium complex

Address correspondence to Abdulaziz U. Joury, MD, Department of Internal Medicine, Ochsner Clinic Foundation, 1514 Jefferson Hwy., New Orleans, LA 70121. Tel: (202) 725-3308. Email: abdulaziz.joury@ochsner.org

\section{INTRODUCTION}

Fungal infections are known for their chronicity and latency and are difficult to eradicate. The pathophysiology of these invading pathogens and the resulting alteration of the immune system are not fully understood. Fungal meningitis is associated with significant rates of morbidity and mortality, especially among immunocompromised patients. Cryptococcosis, considered one of the most important opportunistic infections among patients with human immunodeficiency virus (HIV), has an estimated mortality of $>600,000$ deaths annually worldwide. ${ }^{1,2}$ Among adults with HIV infection, cryptococcal meningitis is the most common form of meningitis, especially in the parts of the world with high HIV seroprevalence. ${ }^{2}$ Immunocompromised patients can present with cryptococcal infection involving almost any organ; however, the organs most commonly affected are the central nervous system and lungs. ${ }^{3-5}$ Symptoms of cryptococcal meningitis include headache, cranial neuropathy, and lethargy, with symptoms of meningeal irritation occurring less frequently. ${ }^{5}$ A patient with HIV and cryptococcosis may present with a focal neurologic deficit and signs of increased intracranial pressure. ${ }^{3-6}$
Cryptococcosis is diagnosed by isolating the clinical specimen from cerebrospinal fluid or detecting the cryptococcal antigen in serum. ${ }^{3}$ Treatment of cryptococcosis has 3 steps: administration of antifungal treatment, management of increased intracranial pressure, and restoration of the immune system upon initiation of antiretroviral therapy. ${ }^{3,4} \mathrm{~A}$ longitudinal study conducted in Zambia showed a 100\% mortality rate among patients with cryptococcal meningitis; death occurred by 7 weeks in a group that did not receive antifungal therapy and by 6 months in a group treated with fluconazole. ${ }^{7}$ The authors concluded that treatment was inadequate. The current treatment recommendation is a 2-week induction phase with amphotericin B and flucytosine to treat cryptococcosis; this combination is considered the most potent regimen. ${ }^{3-5}$

Persistent cryptococcosis is not uncommon, and patients with elevated intracranial pressure and fungal burden have a higher rate of mortality compared to patients with low fungal burden and normal intracranial pressure. ${ }^{8}$ Factors that play a major role in persistent infection include the impaired phagocytosis ability of $T$ cells that extends the survival of Cryptococcus, mechanisms developed by Cryptococcus to 

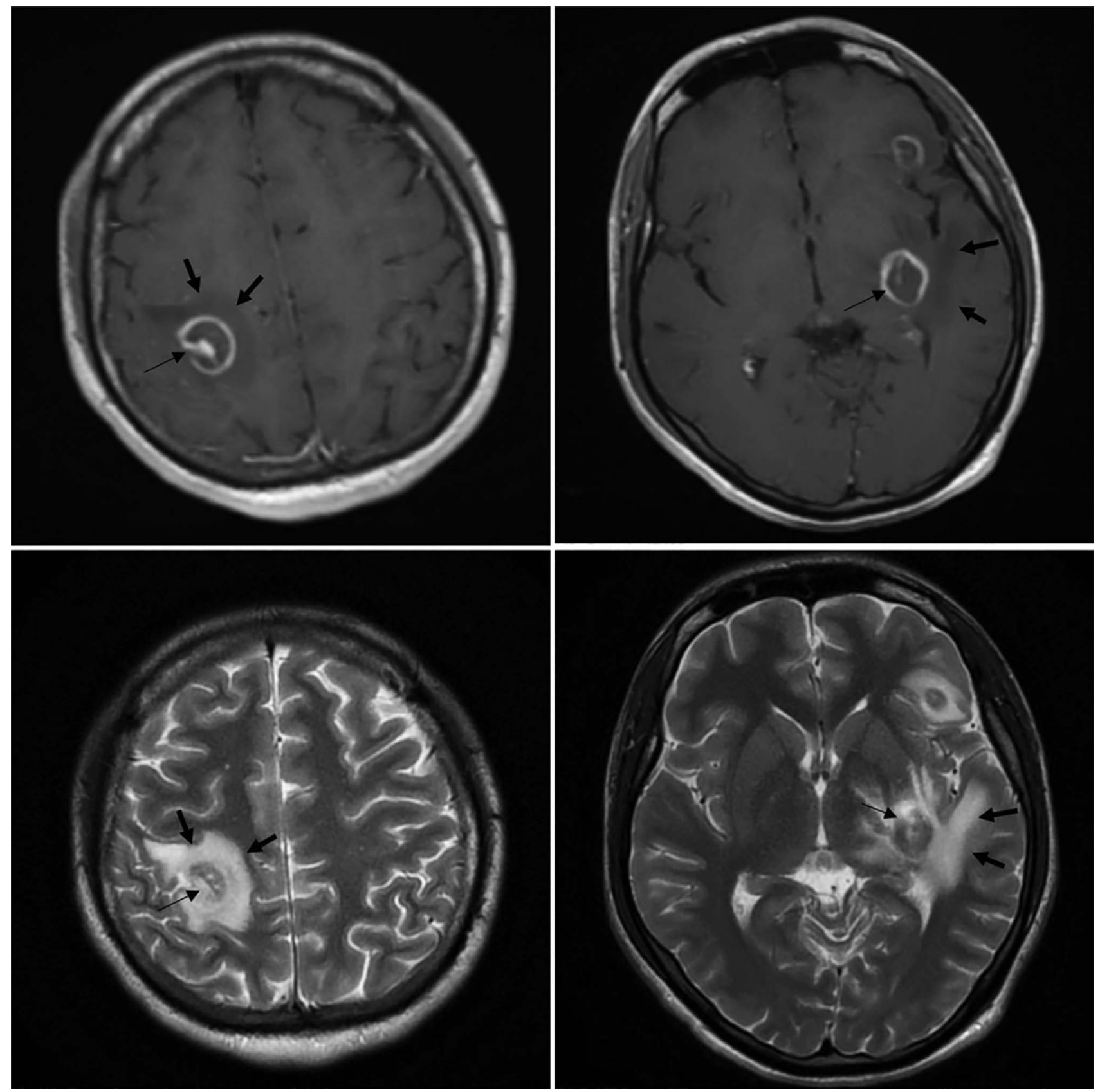

Figure 1. Initial magnetic resonance image of the brain with contrast shows multiple ring-enhancing lesions involving the posterior right parietal lobe, inferior left frontal lobe, and left parietal lobe (thin arrows) with extensive surrounding vasogenic edema signal evident on $\mathrm{T} 1$ and $\mathrm{T} 2$ weighted images (bold arrows).

circumvent host immune cells, and nasal colonization. ${ }^{9-11}$ A study in China found incidence rates of cryptococcosis and Mycobacterium avium complex (MAC) among patients with HIV of $8 \%$ and $11.3 \%$, respectively. ${ }^{12}$ Co-infection with cryptococcosis and MAC is extremely rare even in immunocompromised individuals. We present the case of a 28-yearold male newly diagnosed with HIV who had co-infection of cryptococcosis and MAC.

\section{CASE REPORT}

A 28-year-old male with no significant medical history presented to the emergency department with a 1-month history of headache that he described as band-like. The headache started gradually; became worse during the previous month; and was associated with fever, diplopia, altered sensorium nausea, 10-pound weight loss, productive cough, occasional bloody sputum, and significant night sweats. The patient denied recent travel and intravenous drug abuse. He is a 10-pack-year smoker, is monogamous, and works as a carpenter. Physical examination showed fever and tachycardia, Glasgow Coma Scale score of 15, mild neck stiffness, and intact neurologic examination.

Complete blood count and comprehensive metabolic panel showed normal values except for normocytic anemia. 

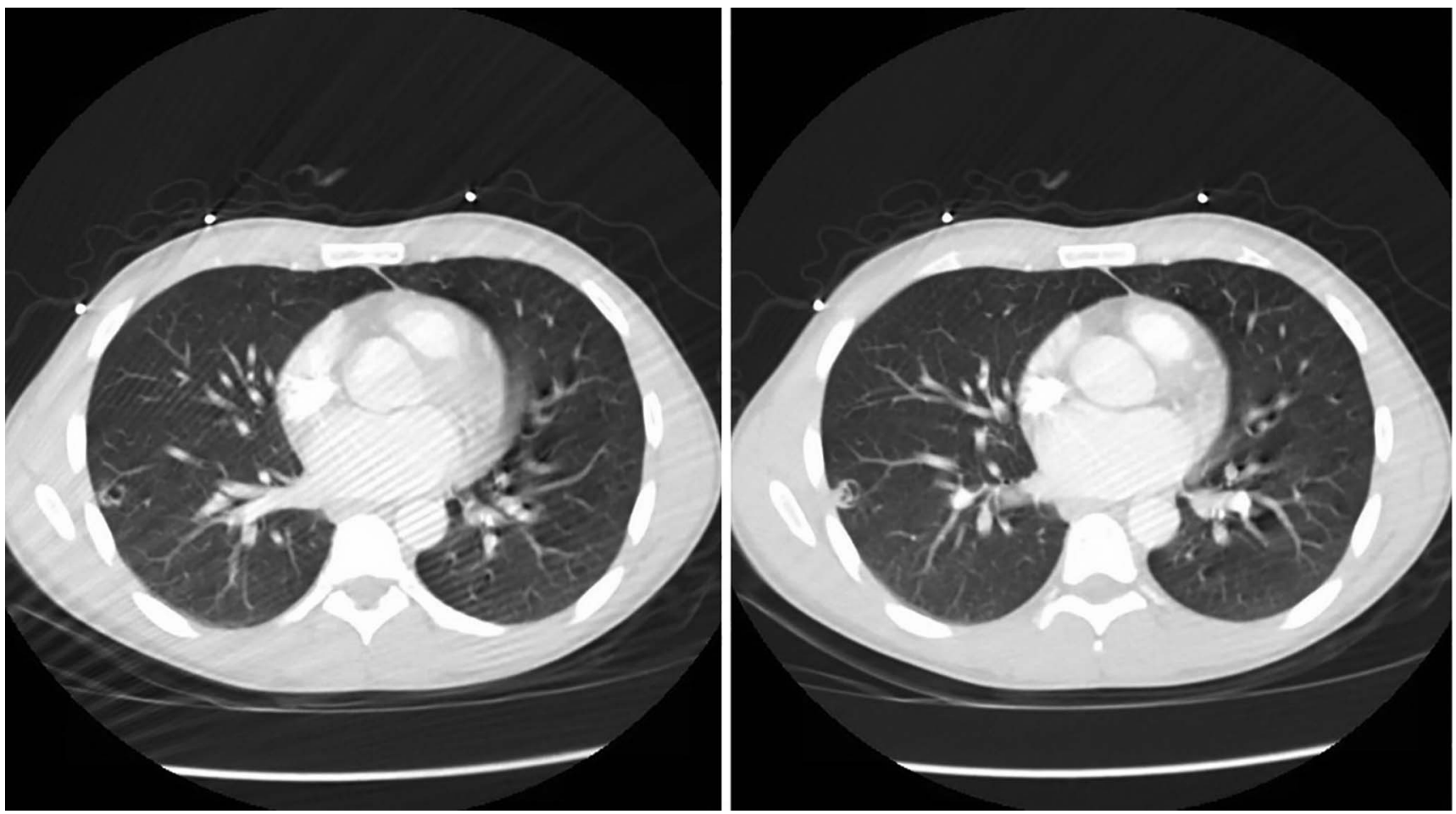

Figure 2. Computed tomography of the chest with contrast shows evidence of a peripheral thin-walled cavitary lesion involving the right lower lobe.

HIV antibodies were positive; the patient's CD4 count was 16 , and viral load was $>500,000$ copies $/ \mathrm{mL}$. Diagnosis of Cryptococcus neoformans infection was confirmed based on positive blood culture; amplified chlamydia DNA and Neisseria gonorrhoeae were negative. Chest x-ray showed mildly prominent central hilar interstitial attenuation without focal consolidation. Brain magnetic resonance imaging (MRI) showed multifocal ring-enhancing lesions with extensive associated vasogenic edema throughout the bilateral convexities (Figure 1). Chest computed tomography scan showed a cavitary-appearing lesion in the right lower lobe peripherally with a relatively thin wall (Figure 2 ).

Treatment with amphotericin B liposome intravenous $300 \mathrm{mg}$ daily and oral flucytosine $1,500 \mathrm{mg} 4$ times daily was initiated. Because of the high index of suspicion that the patient also had toxoplasmosis, oral pyrimethamine 500 mg daily and oral sulfadiazine $1,500 \mathrm{mg} 4$ times daily were started as well. Given the patient's significant vasogenic edema, dexamethasone was initiated with a loading dose of $10 \mathrm{mg}$ followed by a dose of $4 \mathrm{mg} 4$ times daily; the dexamethasone was tapered with a decrease of $50 \%$ of dose per week until complete cessation by the fifth week. For the cavitary lesion of the lung, we adopted watchful waiting given improvement of his cough and night sweats and his ability to breathe without the need for oxygen. Because of the patient's CD4 count, prophylaxis for Pneumocystis pneumonia with oral sulfamethoxazole/trimethoprim 800/160 mg daily and oral azithromycin 1,200 mg weekly were initiated.

Sputum acid-fast bacilli culture was positive for MAC, and oral azithromycin 1,200 mg weekly (administered for 3 weeks) was switched to a daily dose. In addition, oral rifampin $300 \mathrm{mg}$, oral ethambutol $400 \mathrm{mg}$, and oral pyrimethamine $25 \mathrm{mg}$ daily were started with treatment plan intention for 6 months.

Repeat brain MRI 3 weeks after initiation of treatment showed a stable cryptococcal mass and a stable number and distribution of the multiple T2 hyperintense lesions throughout the supratentorial brain parenchyma, with a notable reduction in associated vasogenic edema (Figure 3). Initially, no lumbar puncture had been done in the setting of the original MRI findings; however, 3 weeks after initiation of treatment, lumbar puncture was performed and showed normal opening pressure and normal glucose and protein. The lumbar culture was positive for $C$ neoformans with a positivity of $>1: 256$. Leukemia/lymphoma screen and tests for Mycobacterium tuberculosis DNA by polymerase chain reaction (PCR), Toxoplasma gondii PCR, JC virus DNA PCR, and Epstein-Barr virus PCR were negative.

The patient was hospitalized for 4 weeks. Upon discharge, his medications were adjusted to treat his cryptococcal meningitis, vasogenic edema, and MAC infection. Because the patient had finished his induction phase for cryptococcosis with intravenous amphotericin B and oral flucytosine, therapy with oral fluconazole of $400 \mathrm{mg}$ for 1 dose followed by $200 \mathrm{mg}$ daily for the next 12 weeks was prescribed. For the patient's vasogenic edema during hospitalization, he had received a loading dose of intravenous dexamethasone of $10 \mathrm{mg}$, followed by slow and prolonged tapering during his hospital stay and on discharge. For his MAC infection, the patient was prescribed oral rifampin $300 \mathrm{mg}$ daily, oral ethambutol $400 \mathrm{mg}$ daily, and oral pyrimethamine $25 \mathrm{mg}$ daily with treatment plan intention for 6 months. Follow-up with the infectious disease service and initiation of highly active antiretroviral therapy were planned. 

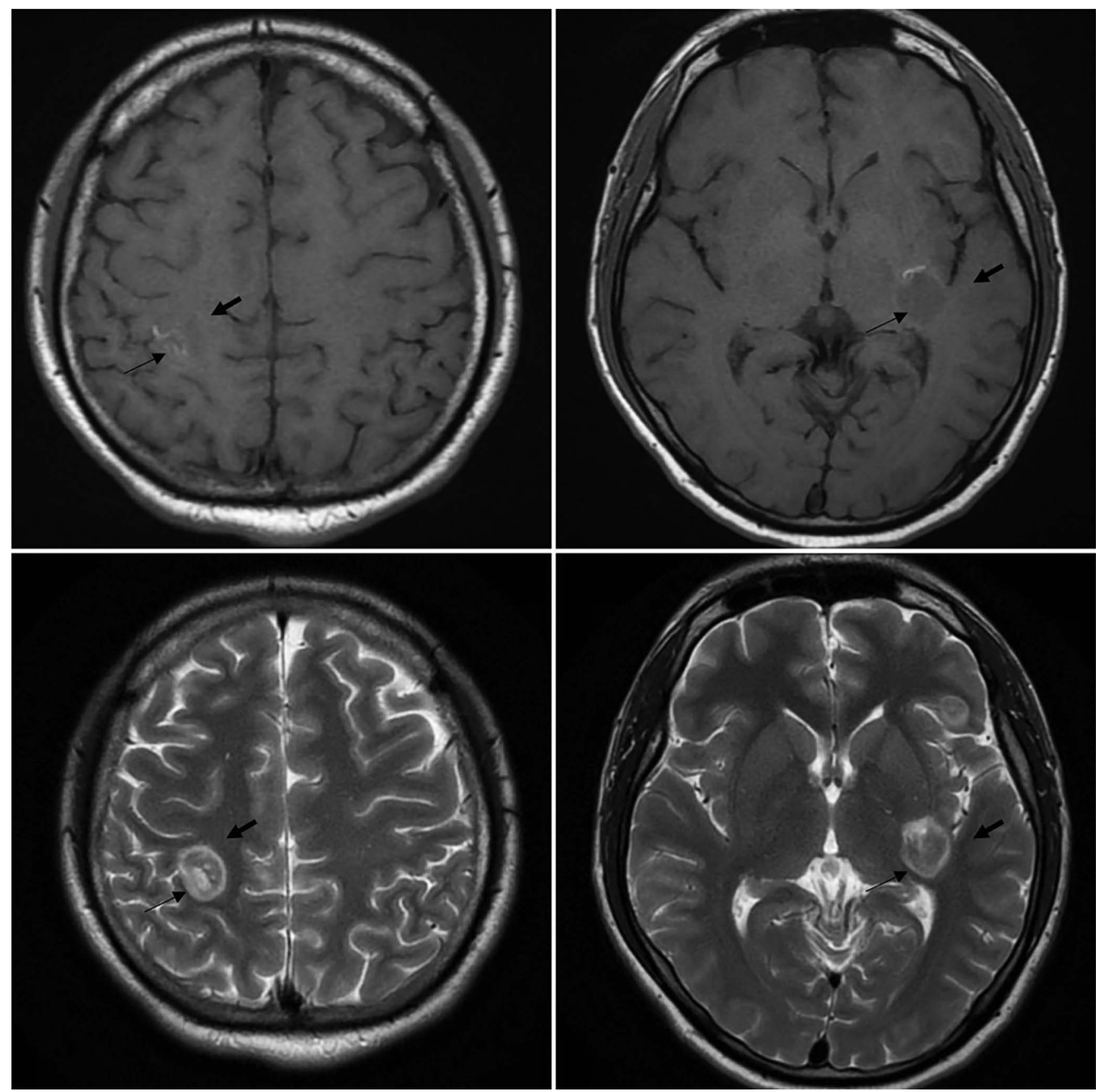

Figure 3. Posttreatment magnetic resonance image of the brain without contrast shows stable multifocal lesions unchanged in size involving the posterior right parietal lobe, inferior left frontal lobe, and left parietal lobe (thin arrows) with significant improvement of surrounding vasogenic edema in comparison to prior imaging; however, the actual lesion remained the same (bold arrows).

\section{DISCUSSION}

Presentation with symptoms such as headache, altered sensorium, and focal neurologic deficit in immunocompromised patients requires immediate investigation to identify life-threatening conditions in this susceptible population. . $^{3,5,6}$ Our patient presented with a 1-month history of headache that persisted despite his taking over-the-counter pain medications. The increase in the severity of his headache during the month might reflect an increase in the size of his brain lesion and the progression of cryptococcosis. Cen- tral nervous system involvement of cryptococcosis is most likely secondary to hematologic spread. ${ }^{3,13}$ In our case, ophthalmic involvement and diplopia were most likely secondary to the increase in intracranial pressure caused by multiple cryptococcal lesions, the presence of the intracranial infection alone ${ }^{14}$ or direct invasion of the fungal pathogen to the ophthalmic neurons.

We delayed starting antiretroviral treatment until after the fifth week in the setting of cryptococcal meningitis. Bisson et al reported that the benefit of an early start of antiretroviral 
treatment is outweighed by potentially serious complications such as immune reconstitution inflammatory syndrome. ${ }^{15}$ Moreover, Boulware et al investigated early antiretroviral treatment initiation (1-2 weeks after diagnosis) and late antiretroviral treatment initiation (5 weeks after diagnosis) among patients who had HIV and cryptococcal meningitis. ${ }^{16}$ Their results showed that mortality among the patients who were treated earlier was significantly higher than in patients who received later antiretroviral treatment initiation (45\% vs $30 \%$; hazard ratio for death, $1.73 ; P=0.03$ ).

Among immunocompromised patients, the differential diagnoses should be expanded to include pulmonary involvement of uncommon bacterial, fungal, or viral infection. Examples of common etiologies of opportunistic lung infections are Mycobacterium species, Pneumocystis jiroveci, and cytomegalovirus. ${ }^{3,4}$

Similarities among these infections can overlap. For example, in pulmonary cryptococcosis, the radiologic finding is not conclusive, and nonspecific findings such as diffuse infiltrates, cavitary lesions, and hilar lymphadenopathy have been described in case reports. ${ }^{17,18}$ In our case, the patient's symptoms of productive cough, night sweats, and significant weight loss coupled with the acid-fast bacilli culture that grew MAC confirmed the diagnosis of MAC infection.

\section{CONCLUSION}

Neurologic symptoms in immunocompromised individuals necessitate immediate medical attention, and radiologic investigation is crucial to monitor treatment response. Cryptococcosis infection is a concern for immunocompromised patients, especially those with HIV, and co-infection with cryptococcosis and MAC is rare. Close clinical follow-up and repeat imaging studies, including brain MRI, to evaluate for persistent disease are highly recommended.

\section{ACKNOWLEDGMENTS}

The authors have no financial or proprietary interest in the subject matter of this article.

\section{REFERENCES}

1. Park BJ, Wannemuehler KA, Marston BJ, Govender N, Pappas PG, Chiller TM. Estimation of the current global burden of cryptococcal meningitis among persons living with HIV/AIDS. AIDS. 2009 Feb 20;23(4):525-530. doi: 10.1097/QAD.0b013e328322ffac.

2. Williamson PR, Jarvis JN, Panackal AA, et al. Cryptococcal meningitis: epidemiology, immunology, diagnosis and therapy. Nat Rev Neurol. 2017 Jan;13(1):13-24. doi: 10.1038/nrneurol.2016.167.

3. Srichatrapimuk S, Sungkanuparph S. Integrated therapy for HIV and cryptococcosis. AIDS Res Ther. 2016 Nov 29;13(1):42. doi: 10.1186/s12981-016-0126-7.

4. Perfect JR, Dismukes WE, Dromer F, et al. Clinical practice guidelines for the management of cryptococcal disease: 2010 update by the infectious diseases society of america. Clin Infect Dis. 2010 Feb 1;50(3):291-322. doi: 10.1086/649858.
5. Panel on opportunistic infections in HIV-infected adults and adolescents. Guidelines for the prevention and treatment of opportunistic infections in HIV-infected adults and adolescents: recommendations from the Centers for Disease Control and Prevention, the National Institutes of Health, and the HIV Medicine Association of the Infectious Diseases Society of America.

https://aidsinfo.nih.gov/contentfiles/lvguidelines/adult_oi.pdf. Accessed October 11, 2018.

6. Bicanic T, Brouwer AE, Meintjes G, et al. Relationship of cerebrospinal fluid pressure, fungal burden and outcome in patients with cryptococcal meningitis undergoing serial lumbar punctures. AIDS. 2009 Mar 27;23(6):701-706. doi: 10.1097/QAD.0b013e32832605fe.

7. Mwaba P, Mwansa J, Chintu C, et al. Clinical presentation, natural history, and cumulative death rates of 230 adults with primary cryptococcal meningitis in Zambian AIDS patients treated under local conditions. Postgrad Med J. 2001 Dec;77(914):769-773.

8. Graybill JR, Sobel J, Saag M, et al. Diagnosis and management of increased intracranial pressure in patients with AIDS and cryptococcal meningitis. The NIAID mycoses study group and AIDS cooperative treatment groups. Clin Infect Dis. 2000 Jan;30(1):47-54.

9. Olszewski MA, Zhang Y, Huffnagle GB. Mechanisms of cryptococcal virulence and persistence. Future Microbiol. 2010 Aug;5(8):1269-1288. doi: 10.2217/fmb.10.93.

10. Anderson DA, Sagha HM. Persistence of infection in mice inoculated intranasally with cryptococcus neoformans. Mycopathologia. 1988 Dec;104(3):163-169.

11. Hospenthal DR, Bennett JE. Persistence of cryptococcomas on neuroimaging. Clin Infect Dis. 2000 Nov;31 (5):1303-1306.

12. Xiao J, Gao G, Li Y, et al. Spectrums of opportunistic infections and malignancies in HIV-infected patients in tertiary care hospital, China. PLoS One. 2013 Oct 25;8(10):e75915. doi: 10.1371/journal.pone.0075915.

13. Portelinha J, Passarinho MP, Almeida AC, Costa JM. Bilateral optic neuropathy associated with cryptococcal meningitis in an immunocompetent patient. BMJ Case Rep. 2014 Jun 11;2014. doi: 10.1136/bcr-2013-203451.

14. Kupfer $C, M c C r a n e ~ E$. A possible cause of decreased vision in cryptococcal meningitis. Invest Ophthalmol. 1974 Oct;13(10):801-804.

15. Bisson GP, Molefi M, Bellamy S, et al. Early versus delayed antiretroviral therapy and cerebrospinal fluid fungal clearance in adults with HIV and cryptococcal meningitis. Clin Infect Dis. 2013 Apr;56(8):1165-1173. doi: 10.1093/cid/cit019.

16. Boulware DR, Meya DB, Muzoora C, et al. Timing of antiretroviral therapy after diagnosis of cryptococcal meningitis. NEngl J Med. 2014 Jun 26;370(26):2487-2498. doi: 10.1056/NEJMoa1312884.

17. Sider L, Westcott MA. Pulmonary manifestations of cryptococcosis in patients with AIDS: CT features. J Thorac Imaging. 1994 Spring;9(2):78-84.

18. Miller WT Jr, Edelman JM, Miller WT. Cryptococcal pulmonary infection in patients with AIDS: radiographic appearance. Radiology. 1990 Jun;175(3):725-728. doi: 10.1148/radiology.175.3.2343121.

This article meets the Accreditation Council for Graduate Medical Education and the American Board of Medical Specialties Maintenance of Certification competencies for Patient Care and Medical Knowledge. 